\title{
Sheared phase-separating binary mixtures with surface diffusion
}

\author{
G. Gonnella ${ }^{1}$ and A. Lamura ${ }^{2, *}$ \\ ${ }^{1}$ Dipartimento di Fisica, Università di Bari, and INFN, \\ Sezione di Bari, Via Amendola 173, 70126 Bari, Italy \\ ${ }^{2}$ Istituto Applicazioni Calcolo, CNR, \\ Via Amendola 122/D, 70126 Bari, Italy
}

(Dated: December 14, 2021)

\begin{abstract}
The phase-separation process of a binary mixture with order-parameter-dependent mobility under shear flow is numerically studied. The ordering is characterized by an alternate stretching and bursting of domains which produce oscillations in the physical observables. The amplitude of such modulations reduce in time when the mobility vanishes in the bulk phase, disfavoring the growth of bubbles coming from bursted domains. We propose two equations for the typical sizes $R_{x}$ and $R_{y}$ of domains finding the long-time behaviors $R_{x} \sim t^{5 / 4}$ and $R_{y} \sim t^{1 / 4}$ in the flow and shear directions, respectively, in the case of surface diffusion. A reduction of the excess viscosity with increasing shear rate is observed in simulations.

PACS numbers: 64.75.Gh Phase separation and segregation in model systems; 83.50.Ax Steady shear flow; 02.70.-c Computational techniques, simulations
\end{abstract}

\footnotetext{
* Corresponding author: antonio.lamura@cnr.it
} 


\section{INTRODUCTION}

The phase separation process when a mixture made of two components is suddenly quenched below the critical temperature is a well studied and understood phenomenon 1]. Immediately after the quench, coherent regions rich in either of the phases form and grow. Typically, if scaling holds, these domains can be characterized in terms of a single time-dependent length scale $R(t)$ which grows at late times with a power law $R(t) \sim t^{\alpha}$, where $t$ is time. The problem is very interesting and attracts a lot of interest [2 10].

In the case of binary mixtures with constant mobility, the value of the exponent $\alpha$ depends on some general properties of the considered system. For a nonconserved scalar order parameter, as in ferromagnets, it results to be $\alpha=1 / 2[1]$. When the order parameter is conserved and hydrodynamic effects are ignored, is found $\alpha=1 / 3[11]$. External shear flow is known to modify deeply phase separation producing elongated domains as in simulations [12, 13] and experiments [14 16]. Numerical solutions of the scalar order parameter dynamics [17] as well as analytical [18] and numerical calculations [19] in the large- $N$ limit of an $N$-vector order parameter, which corresponds to the self-consistent approximation of the scalar case, gave confirmation of the existence of anisotropy in the growing domains with two characteristic lengths and two different exponents. The growth exponent is not affected in the shear direction perpendicular to the flow while in the large- $N$ limit the exponent along the flow direction is increased by 1 [18, 19]. Moreover, all the physical observables are modulated by oscillations on a logarithmic time scale which are related to a cyclic mechanism of storage and dissipation of elastic energy.

A more realistic phase separation scenario, that takes into account the diffusion of molecules of different species along interfaces between domains of different composition, can be obtained by considering an order-parameter-dependent mobility $\Gamma(\varphi) \propto\left[1-c(T) \varphi^{2}\right]$ [20] where $\varphi$ is the difference of concentrations of the two components of the mixture. The term $c(T) \rightarrow 1$ for temperature $T \rightarrow 0$ and $c(T) \rightarrow 0$ for $T \rightarrow T_{c}, T_{c}$ being the critical temperature. This form of the mobility is such that in the case of a deep quench $(T \rightarrow 0)$ the diffusion in the bulk, where $\varphi \simeq 1$, is suppressed while it is favored along interfaces where $\varphi \simeq 0$. This latter mechanism, known as surface diffusion (SD), has growth exponent $\alpha=1 / 4$ [21] and has relevant technological and scientific applications, for example, in deep quenches of polymer mixtures [22] and coarsening of porous structures by surface diffusion 
[23]. On the other hand, for shallow quenches $\left(T \lesssim T_{c}\right)$ the mobility stays constant and phase separation proceeds by the Lifshitz-Slyozov mechanism [24] with the aforementioned growth exponent $\alpha=1 / 3$. In this case the growth of domains is related to bulk diffusion (BD), since molecules of one component evaporate from more curved interfaces and diffuse through the bulk of the other component diminishing the curvature of the interface. We add for completeness that in the case of asymmetric mixtures the minority phase forms isolated droplets. These collide due to Brownian motion and coalesce with the same growth exponent of the Lifshitz-Slyozov mechanism [25]. Numerical studies of phase separation in binary mixtures with field-dependent mobility found confirmation of the exponent $\alpha=1 / 4$ for $c=1$ as expected for a scalar order parameter [22, 26-30]. For intermediate values $0<c<1$ there is a crossover between $\alpha=1 / 4$ and $\alpha=1 / 3$. In the case of an $N$-dimensional order parameter with mobility given by $\Gamma(\boldsymbol{\varphi})=1-c \boldsymbol{\varphi}^{2} / N$ simulations gave $\alpha=1 / 6$ for $c=1$ and a crossover from $\alpha=1 / 6$ to $\alpha=1 / 4$ for $0<c<1$ with $N=2,3,4$ [31]. We remark that the diffusive exponent of a vectorial order parameter is also $1 / 4$, due to a different physical mechanism form that of SD. A more general form $\Gamma(\varphi)=\left[1-\varphi^{2} / N\right]^{\beta}$ with $\beta>0$ has been also introduced and solved in the scalar case $N=1$ [32] and in the large- $N$ limit [33]. Effects of shear flow have been considered in the large- $N$ limit for this latter form of the mobility that includes the standard case with $\beta=1$. It is found that domains grow with characteristic length $t^{(2 \beta+5) / 2(\beta+2)}$ along the flow direction and $t^{1 / 2(\beta+2)}$ along the shear direction with logarithmic corrections [34] and oscillations of the physical observables appear to be damped [35]. However, domain morphology during phase separation cannot by fully outlined when using the large- $N$ limit since interfaces are missed in the vectorial model [11]. For this reason we want to consider the effects of shear flow on the phase separation process in the case of a scalar order parameter with field-dependent mobility by solving numerically the phenomenological equation of the order parameter in two spatial dimensions. The novelty of the present study relies on addressing for the first time the effects of surface diffusion in the simulation of a phase separating binary mixture under shear.

The paper is organized as follows. Section II describes the model and Sect. III is devoted to present and discuss numerical results. Phenomenological equations for the typical sizes of domains suggest domain growth with power laws $t^{5 / 4}$ and $t^{1 / 4}$ in the flow and shear directions, respectively. Simulations give compatible results and provide direct evidence of a cyclic stretching and bursting of domains which are responsible of oscillations in the physical 
observables. The amplitude reduces in time due to the vanishing of the mobility in the bulk phase which makes difficult the growth of bubbles coming from bursted domains thus finding confirmation of the argument put forward in Ref. [35]. Finally, we draw conclusions.

\section{THE MODEL}

The equilibrium properties of the system we are considering, are described by the Ginzburg-Landau free-energy

$$
\mathcal{F}\{\varphi\}=\int d \boldsymbol{r}\left\{-\frac{a}{2} \varphi^{2}+\frac{b}{4} \varphi^{4}+\frac{\kappa}{2}|\nabla \varphi|^{2}\right\}
$$

where $\varphi$ is the order parameter. As usual, it is assumed $b>0$ ensuring stability. The coefficient $a=\left(T_{c}-T\right) / T_{c}$ can be considered as a reduced temperature where $T$ is the temperature of the system and $T_{c}$ is the critical value below which the fluid is ordered. When $T<T_{c}$ the polynomial terms of the free-energy density have two symmetric minima located at $\varphi_{e q}(T)= \pm \sqrt{a / b}$ which are the equilibrium values of the order parameter. Finally, the parameter $\kappa>0$ encodes the energy cost for the creation of interfaces between domains of different composition. The chemical potential difference between the two components can be computed from Eq. (11) and is given by

$$
\mu=\frac{\delta \mathcal{F}}{\delta \varphi}=-a \varphi+b \varphi^{3}-\kappa \nabla^{2} \varphi
$$

The time evolution of the order parameter $\varphi$ is described by the convection-diffusion equation

$$
\frac{\partial \varphi}{\partial t}+\nabla \cdot(\varphi \boldsymbol{v})=\nabla \cdot[\Gamma(\varphi) \nabla \mu]
$$

The convective term on the l.h.s. couples $\varphi$ to the external velocity field which is given here by a linear shear flow $\boldsymbol{v}=\dot{\gamma} y \boldsymbol{e}_{x}$ where $\dot{\gamma}$ is the shear rate, $y$ the coordinate along the $y$-direction (shear direction), and $\boldsymbol{e}_{x}$ the unit vector along the $x$-direction (flow direction). We assume the fluid to be very viscous so that the Reynolds number (ratio of inertial to viscous forces) is very low and the capillary number (ratio of viscous to interfacial (in two dimensions) forces) is very large. In this way hydrodynamic effects are neglected in the present model so that Eq. (3) is not coupled to the Navier-Stokes equation for the flow field. Very recently the general case, when the capillary number is not large, has been considered in Ref. [36] for the phase separation of sheared binary mixtures. We also neglect thermal 
fluctuations. The mobility depends explicitly on the order parameter in order to have a more realistic description of the phase separation, especially for polymer mixtures [20], and is given by

$$
\Gamma(\varphi)=\Gamma_{0}\left[1-\varphi^{2} / \varphi_{e q}^{2}(0)\right]
$$

where $\Gamma_{0}$ is a constant. We comment here about the role of the temperature on the mobility when there is no external flow $(\boldsymbol{v}=0)$. In the case of a deep quench $(T \rightarrow 0)$, the diffusion in the bulk, where $\varphi \simeq \varphi_{e q}$, is suppressed since $\Gamma\left(\varphi_{e q}(T)\right) \rightarrow 0$ promoting surface diffusion. On the other hand for shallow quenches $\left(T \rightarrow T_{c}\right)$, the mobility is not significantly reduced in the bulk since the $\varphi_{e q}^{2}(T) \ll \varphi_{e q}^{2}(0)$. In this limit the Cahn-Hilliard equation [37] is recovered whose numerical solution [38] gives evidence of the growth exponent $\alpha=1 / 3$ typical of bulk diffusion.

Equation (31) can be written in a dimensionless form [38] after redefining time, space, and field scales by $\tau=\kappa /\left(2 \Gamma_{0} a^{2}\right), \xi=\sqrt{\kappa / a}$, and $\varphi_{e q}(T)$, respectively. In the following the symbol ^ will denote dimensionless quantities. The only relevant parameters in the dimensionless equation are the mobility and the shear rate. The mobility assumes the form $\hat{\Gamma}(\hat{\varphi})=1-\lambda \hat{\varphi}^{2}$, where $\lambda=\varphi_{e q}^{2}(T) / \varphi_{e q}^{2}(0)$ goes from 0 to 1 when the temperature is reduced from $T_{c}$ to 0 , the bulk equilibrium values of the order parameter are \pm 1 , and the shear rate is $\hat{\dot{\gamma}}=\dot{\gamma} \tau$.

\section{NUMERICAL RESULTS AND DISCUSSION}

We have simulated Eq. (3) in two dimensions by using a finite-difference scheme. The field $\varphi(\boldsymbol{r}, t)$ is discretized on the nodes $\left(x_{i}, y_{j}\right)(i, j=1,2, \ldots, L)$ of a square lattice with space step $\Delta x$ and $L \times L$ nodes. Time is discretized in time steps $\Delta t$. Periodic boundary conditions were implemented in the $x$-direction while Lees-Edwards boundary conditions [39] were used in the normal direction. The latter conditions require the identification of a point on the lower row at $\left(x_{i}, y_{1}\right)$ with the one located on the upper row at $\left(x_{i}+\dot{\gamma} L \Delta t, y_{L}\right)$ $(i=1,2, \ldots, L)$ to take into account the space shift due to shear. The time derivative in Eq. (3) was implemented by using an explicit first-order Euler algorithm [38] while standard central difference schemes were adopted for spatial derivatives appearing in the convective and diffusion terms [40].

Simulations were run using lattices with $L=1024$. We fix $a=b=\kappa=\Gamma_{0}=1$ and write 
the mobility in Eq. (3) as $\Gamma(\varphi)=1-\lambda \varphi^{2}$ with $0 \leq \lambda \leq 1$. This form of the mobility allows us to consider a deep quench $(\lambda=1)$ where SD is the leading diffusion mechanism. In this case the numerical model can become unstable when $|\varphi|>1$, due to numerical fluctuations, since the mobility results to be negative. To overcome this problem we used a fine mesh with $\Delta x=0.5,1$ and a very small time step $\Delta t=10^{-3}$ enforcing the value $1(-1)$ to $\varphi$ whenever $\varphi>1(\varphi<-1)$. Neither relevant differences were observed for the two values of $\Delta x$ nor significant violation of field conservation could be appreciated. Equation (3) was also solved by using intermediate values $\lambda<1$. The limit case $\lambda=0$ allows the simulation of a quench where BD dominates the phase separation process. In this way it was possible to consider the crossover between surface and bulk diffusion mechanisms under the presence of shear. The system was prepared in a disordered state $\left(T>T_{c}\right)$ with $\langle\varphi\rangle=0$ corresponding to a symmetric composition of the mixture, $\langle\ldots\rangle$ denoting an average over the system. The results here exposed were obtained with $\Delta x=1, \dot{\gamma}$ in the range $\left[10^{-3}, 5 \times 10^{-2}\right], \lambda=0,0.4,0.8,1$, and averaging over five independent realizations of the system. All the quantities in the following are expressed in units of $\Delta x, \Delta t$, and $\varphi_{e q}$.

The morphology of the phase-separation process in the SD case $(\lambda=1)$ is shown for $\dot{\gamma}=5 \times 10^{-2}$ in Fig. 1 at consecutive values of the strain $\dot{\gamma} t$ on a portion of the whole lattice of size $256 \times 256$. At the beginning a bicontinuous structure is formed yet with no appreciable deformation induced by shear. For values $\dot{\gamma} t>1$ the external flow starts to modify domains which are elongated, tilted, and then bursted by shear when accumulated stress overcomes surface tension. At $\dot{\gamma} t=7$ it can be noted that domains are stretched by the flow and characterized by different thicknesses. At later times the flow further deforms domains which may break up, due to the aforementioned mechanism, giving rise to several bubbles which spread all over the system $(\dot{\gamma} t=16)$. In the SD case the suppression of bulk diffusion inhibits the growth of these small bubbles originating from the shear-induced bursting of domains. As a matter of comparison we plot in Fig. 2 the configurations at the same value of the strain for the values $\lambda=0,0.8$. It is evident that by decreasing $\lambda$, the number of bubbles in the system reduces being minimum in the case with $\lambda=0$ where bubbles can grow by bulk diffusion. This feature will be further discussed in the following. As a consequence, bubbles cannot be further deformed in the SD case by the flow but can eventually merge with larger domains when these are stretched again by the flow and reach some bubble $(\dot{\gamma} t=24)$. In the large- $N$ limit [35] it was found that the phenomenon of 
elongation and bursting of domains is cyclic. Here we find an indication of this periodic behavior but the finite size of the computational domain does not allow the observation on very long times to find full evidence of periodic behavior on a logarithmic time scale.

In order to characterize the size distribution of growing domains, the normalized probability distributions functions $P\left(L_{x, y}\right)$ of finding domains of lengths $L_{x}$ and $L_{y}$ along the flow and the shear directions, respectively, were computed. First we estimated the extensions $L_{x}$ of the unidimensional domains of different composition along all the rows of the computational mesh. From the recorded values of $L_{x}$, we finally computed $P\left(L_{x}\right)$. The same procedure was followed along the shear direction to compute $P\left(L_{y}\right)$. The plots of $P\left(L_{x}\right)$ and $P\left(L_{y}\right)$ are shown in Fig. 3 at different times with $\lambda=1$. At $\dot{\gamma} t=1$ both $P\left(L_{x}\right)$ and $P\left(L_{y}\right)$ have a single main peak at $L_{x 1} \simeq L_{y 1} \simeq 5$, corresponding to initial isotropic domains, and a small shoulder in the tail. Later anisotropy effects become evident in the distributions. Along the flow direction it is possible to observe at $\dot{\gamma} t=7$ the formation of a second peak at $L_{x 2} \simeq 20$, corresponding to more elongated domains, which prevails on the other one at $L_{x 1}$. Along the shear direction, at the same time, the main peak decreases in height while the distribution broadens corresponding to the presence of domains with different thicknesses. The further stretching of domains is such that the peak at $L_{x 2}$ moves at following time towards a larger value $(\simeq 40)$ while the breaking of elongated domains, which promotes the formation of bubbles, is such that the peak at $L_{x 1}$ dominates (see $\dot{\gamma} t=16$ in Fig. 3). This supports the previously described picture where one can observe an alternate dominance of elongated and bursted domains. At the same time the peak of $P\left(L_{y}\right)$ at $L_{y 1}$ grows again while the tail decays more rapidly with $L_{y}$ than at the previous time as consequence of the formation of bubbles of typical size $L_{x 1} \simeq L_{y 1}$. At the last time considered in Fig. 3) the peak at $L_{x 1}$ of $P\left(L_{x}\right)$ still grows while the second peak moves at $L_{x 2} \simeq 60$, since domains continue to be stretched, decreasing in height and broadening. The distribution $P\left(L_{y}\right)$ does not change significantly. Figure 4 compares the probability distribution functions in the BD and SD cases. It is found that the peak at $L_{x 1}$ is always higher in the SD case for $\dot{\gamma} t>10$ while in the $\mathrm{BD}$ case there is an alternate dominance of the peaks at $L_{x 1}$ and $L_{x 2}$. This confirms the larger abundance of bubbles which cannot grow after their formation since the variable mobility suppresses diffusion in the bulk. Therefore bubbles cannot be elongated too much by the flow and this also reflected in the distribution $P\left(L_{y}\right)$. Indeed its peak at $L_{y 1}$ does not change significantly in height for $\dot{\gamma} t>10$ while in the BD case the peak shows 
weak oscillations since domains are continuously stretched and bursted while growing.

In the isotropic case without shear it was proposed the following equation for the domain size $R(t)[26]$ :

$$
\frac{d R(t)}{d t}=(1-\lambda) \frac{A}{R^{2}}+\lambda \frac{B}{R^{3}}
$$

where the first and second terms on the r. h. s. take into account the bulk and surface diffusion, respectively, $A$ and $B$ being two constants. It comes out that $R(t)$ grows in time with a power law with exponents $\alpha=1 / 4$ for $\lambda=1$ and $\alpha=1 / 3$ for $\lambda=0$. A crossover between these two regimes was found in numerical simulations for intermediate values of $\lambda$ [26]. In the case with shear, denoting by $R_{x}$ and $R_{y}$ the sizes of an elongated domain along the flow and the shear directions, respectively, Eq. (5) can be generalized to:

$$
\begin{aligned}
& \frac{d R_{x}(t)}{d t}=C_{0} \dot{\gamma} R_{y}+(1-\lambda) C_{1}\left(\frac{1}{R_{x}^{2}}+\frac{1}{R_{y}^{2}}\right)+\lambda C_{2}\left(\frac{1}{R_{x}^{3}}+\frac{1}{R_{y}^{3}}\right) \\
& \frac{d R_{y}(t)}{d t}=(1-\lambda) C_{1}\left(\frac{1}{R_{x}^{2}}+\frac{1}{R_{y}^{2}}\right)+\lambda C_{2}\left(\frac{1}{R_{x}^{3}}+\frac{1}{R_{y}^{3}}\right)
\end{aligned}
$$

where $C_{0}, C_{1}$, and $C_{2}$ are constants. The first term on the r.h.s. of Eq. (6) takes into account the growth along the $x$-direction caused by the advection of domains due to the shear and is related to the amount of flow intercepted by the domain which is proportional to $\dot{\gamma} R_{y}$. Solving the equations under the hypothesis $R_{x} \gg R_{y}$ at long times, we find the asymptotic behaviors $R_{x} \sim \dot{\gamma} t^{5 / 4}$ and $R_{y} \sim t^{1 / 4}$ with $\lambda=1$ and recover the results $R_{x} \sim \dot{\gamma} t^{4 / 3}$ and $R_{y} \sim t^{1 / 3}$ with $\lambda=0$ [17]. The average sizes $R_{x}$ and $R_{y}$ of domains were computed in simulations in two different ways. By measuring the total lengths $I_{x}$ and $I_{y}$ of the interfaces between domains along the flow and the shear directions, respectively, we defined $R_{x, y}=L^{2} / I_{x, y}$. The second procedure relies on the use of the structure factor $C(\boldsymbol{k}, t)=\langle\varphi(\boldsymbol{k}, t) \varphi(-\boldsymbol{k}, t)\rangle$, where $\varphi(\boldsymbol{k}, t)$ is the Fourier transform of the order parameter. From this quantity, the average sizes of domains were computed as

$$
R_{x, y}^{\prime}(t)=\pi \frac{\int d \boldsymbol{k} C(\boldsymbol{k}, t)}{\int d \boldsymbol{k}\left|k_{x, y}\right| C(\boldsymbol{k}, t)} .
$$

The time behavior of $R_{x}$ and $R_{y}$ is reported in Fig. 5 for $\lambda=0,0.4,0.8,1$. We found that $R_{x}^{\prime}$ and $R_{y}^{\prime}$ have time dependencies similar to the ones of $R_{x}$ and $R_{y}$ being $R_{x, y}^{\prime}(t) \simeq R_{x, y}(t) / 3$ over the explored time range. It can be seen that $R_{x} \simeq R_{y}$ until $\dot{\gamma} t \simeq 1$, independently on $\lambda$, while later $R_{x}$ grows faster than $R_{y}$. We find that the growth of $R_{x}$ at long times is slower when increasing $\lambda$ as expected from the asymptotic solution of Eq. (66). However, 
the limited size of the simulated system does not allow us to have a reliable estimate of the growth exponent along the flow direction. The size $R_{y}$ along the shear direction shows oscillations which are related to the alternate stretching (minimum of $R_{y}$ ) and breaking (maximum of $R_{y}$ ) of domains. The amplitude of such oscillations shrinks when increasing $\lambda$. In the case of surface diffusion $(\lambda=1)$ oscillations appears to reduce significantly in time. The same behavior was observed in the large- $N$ limit [35] where all the physical observables showed damped oscillations on a logarithmic time-scale. In that model it was guessed that the damping of oscillations might have been related to the suppression of bulk diffusion. In this paper, we directly show that bubbles, coming from the bursting of overstretched domains, cannot be elongated too much by the flow. As a consequence, we observe less wide oscillations which further reduce in time since the mechanism of growth inhibition becomes more effective. Indeed we find evidence, as illustrated in Figs. 1 and 3, of this picture. However, the period of observation, due to the finite extension of the simulated system, is limited to a bit more than one cycle of stretching and bursting.

Of experimental interest is the excess viscosity which measures the variation of the mixture viscosity with respect to the homogeneous case. It is defined as [41]

$$
\Delta \eta=-\frac{1}{\dot{\gamma}} \int \frac{d \boldsymbol{k}}{(2 \pi)^{2}} k_{x} k_{y} C(\boldsymbol{k}, t)
$$

whose time behavior is shown in Fig. 6 for different values of $\lambda$. We find that the excess viscosity grows oscillating reaching a global maximum at $\dot{\gamma} \simeq 8$ when domains are stretched. Later $\Delta \eta$ decays to zero due to the dissipation of the energy stored by elongated domains when they start to burst after further stretching. It appears that the excess viscosity shows, when increasing $\lambda$, reduced oscillations in the long time limit, due to the inhibition of the growth of circular domains coming from bursting, and a slower time decay. In the SD case the behavior of the excess viscosity in the long time limit can be obtained by assuming that scaling is verified asymptotically. This implies that the structure factor can be written in the anisotropic case as $C(\boldsymbol{k}, t) \sim R_{x} R_{y} f\left(k_{x} R_{x}, k_{y} R_{y}\right)$ [17]. Then it can be shown that the excess viscosity $\Delta \eta \sim 1 /\left(\dot{\gamma} R_{x} R_{y}\right)$ has asymptotic behavior $\Delta \eta \sim \dot{\gamma}^{-1 / 2}(\dot{\gamma} t)^{-3 / 2}$ where the exponent $-3 / 2$ is, indeed, smaller than the value $-5 / 3$ valid in the $\mathrm{BD}$ case [17]. However, present simulations do not allow a reliable estimate of the decay exponent.

Finally, the values $(\Delta \eta)_{M}$ of the excess viscosity at its maximum are plotted as a function of the shear rate in Fig. 7 in the SD case. A reduction of $(\Delta \eta)_{M}$ can be seen and can 
be understood in the following way. The previous scaling analysis of the excess viscosity behavior suggests that, for a fixed value of the strain $\dot{\gamma} t$, the scaling $\Delta \eta \sim \dot{\gamma}^{-1 / 2}$ should hold. We find in simulations an effective exponent -0.9 which is smaller than the expected one.

\section{CONCLUSIONS}

In this paper we have studied numerically the phase separation of a binary mixture under shear flow when the mobility depends explicitly on the order parameter. The morphology of patterns has been analyzed and discussed by computing the probability distribution functions of the size of domains along the two spatial directions. When surface diffusion dominates the coarsening of the mixture, it is found that domains are elongated, tilted, and finally bursted by the flow. This process repeats in time producing oscillations in physical observables as in the case with constant mobility where bulk diffusion is the leading mechanism. Here the novelty is that bubbles, coming from the disruption of stretched domains, cannot grow due to the reduction of the diffusion in the bulk phase when surface diffusion prevails. As a consequence, the oscillation amplitudes in the size of domains as well as in the excess viscosity reduce in time going from the bulk to the surface diffusion. This gives a direct evidence of the picture put forward in the large- $N$ limit [35]. A generalization of a phenomenological equation for the typical size of domains has been proposed. This allows us to obtain the asymptotic behaviors of characteristic measures of growing patterns along the flow and the shear directions. Numerical simulations show that the growth slows down when going from bulk diffusion to surface diffusion. The same behavior is also observed for

the excess viscosity whose maximum value, at fixed strain, decreases with increasing shear rate.

[1] J.D. Gunton, M. San Miguel, and P. Sahni, in Phase Transition and Critical Phenomena, ed. by C. Domb and J.H. Lebowitz (Academic, London, 1983), Vol. 8.

[2] H. Tanaka, J. Phys.: Condens. Matter 12, R207 (2000).

[3] G. Gonnella, and A. Lamura, and A. Piscitelli, J. Phys. A 41, 105001 (2008).

[4] A. Singh, A. Mukherjee, H. M. Vermeulen, G. T. Barkema, and S. Puri, J. Chem. Phys. 134, 044910 (2011). 
[5] G. Gonnella, A. Lamura, A. Piscitelli, and A. Tiribocchi, Phys. Rev. E 82, 046302 (2010).

[6] P. Thareja, J. Rheol. 55, 1375 (2011).

[7] S. Roy and S. K. Das, Soft Matter 9, 4178 (2013).

[8] S. Roy and S. K. Das, J. Chem. Phys. 139, 044911 (2013).

[9] J. Jung, E. Jang, M. A. Shoaib, K. Jo, and J. S. Kim, J. Chem. Phys. 144, 134502 (2016).

[10] T. Tsukada and R. Kurita, Phys. Rev. Research 2, 013382 (2020).

[11] J. Bray, Adv. Phys. 43, 357 (1994).

[12] T. Ohta, H. Nozaki, and M. Doi, Phys. Lett. A 145, 304 (1990); J. Chem. Phys. 93, 2664 (1990).

[13] D. H. Rothman, Europhys. Lett. 14, 337 (1991).

[14] T. Hashimoto, K. Matsuzaka, E. Moses, and A. Onuki, Phys. Rev. Lett. 74, 126 (1994).

[15] C. C. Han, Y. H. Yao, R. Y. Zhang, and E. K. Hobbie, Polymer 47, 3271 (2006).

[16] D. Derks, D. G. A. L. Aarts, D. Bonn, A. Imhof, J. Phys.: Condens. Matter 20, 404208 (2008).

[17] F. Corberi, G. Gonnella, A. Lamura, Phys. Rev. Lett. 83, 4057 (1999); Phys. Rev. E 62, 8064 (2000).

[18] N. P. Rapapa and A. J. Bray, Phys. Rev. E 83, 3856 (1999).

[19] F. Corberi, G. Gonnella, A. Lamura, Phys. Rev. Lett. 81, 3852 (1998); Phys. Rev. E 61, 6621 (2000).

[20] J. S. Langer, M. Bar-on, and H. D. Miller, Phys. Rev. A 11, 1417 (1975); K. Kitahara and M. Imada, Prog. Theor. Phys. Suppl. 64, 65 (1978).

[21] H. Furukawa, Adv. Phys. 34, 703 (1985).

[22] J. K. Wolterink, G. T. Barkema, and S. Puri, Phys. Rev. E 74, 011804 (2006).

[23] P.-A. Geslin, M. Buchet, T. Wada, and H. Kato, Phys. Rev. Mat. 3, 083401 (2019).

[24] I. M. Lifshitz and V. V. Slyozov, J. Phys. Chem. Solids 19, 35 (1961).

[25] K. Binder and D. Stauffer, Phys. Rev. Lett. 33, 1006 (1974).

[26] A. M. Lacasta, A. Hernandez-Machado, J. M. Sancho, and R. Toral, Phys. Rev. B 45, 5276 (1992).

[27] S. Puri, A. J. Bray, and J. L. Lebowitz, Phys. Rev. E 56, 758 (1997).

[28] J. Zhu, L.-Q. Chen, J. Shen, and V. Tikare, Phys. Rev. E 60, 3564 (1999).

[29] C. Castellano and F. Corberi, Phys. Rev. E 61, 3252 (2000).

[30] S. van Gemmert, G. T. Barkema, and S. Puri, Phys. Rev. E 72, 046131 (2005). 
[31] F. Corberi and C. Castellano, Phys. Rev. E 58, 4658 (1998).

[32] A. J. Bray and C. L. Emmott, Phys. Rev. B 52, R685 (1995).

[33] C. L. Emmott and A. J. Bray, Phys. Rev. E 59, 213 (1999).

[34] N. P. Rapapa, Phys. Rev. E 61, 247 (2000).

[35] G. Gonnella, A. Lamura, and D. Suppa, Eur. Phys. J. B 13, 169 (2000).

[36] Chih-Che Chueh, A. Bertei, and R. Mauri, Phys. Fluids 32, 023307 (2020).

[37] J. W. Cahn and H. E. Hilliard, J. Chem. Phys. 28, 258 (1958).

[38] T. M. Rogers, K. R. Elder, and R. C. Desai, Phys. Rev. B 37, 9638 (1988).

[39] A. W. Lees and S. F. Edwards, J. Phys. C 5, 1921 (1972).

[40] J. C. Strikwerda, Finite Difference Schemes and Partial Differential Equations (Chapman and Hall, New York, 1989).

[41] A. Onuki, J. Phys. Condens. Matter 9, 6119 (1997). 

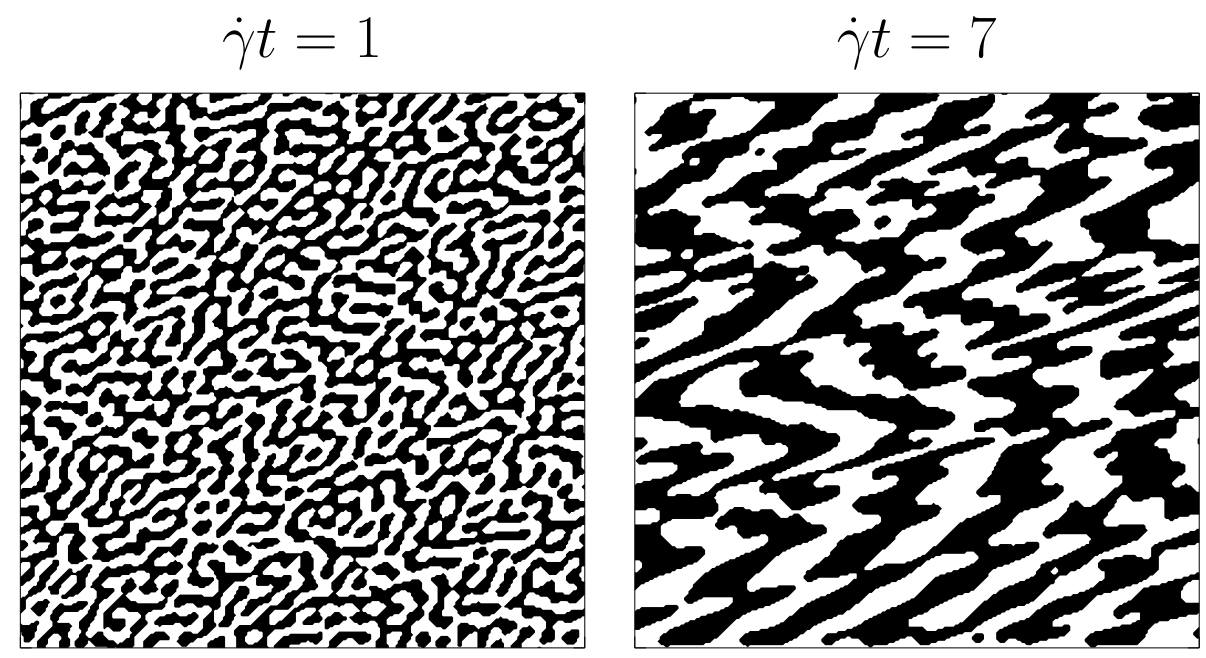

$\dot{\gamma} t=16$

$\dot{\gamma} t=24$
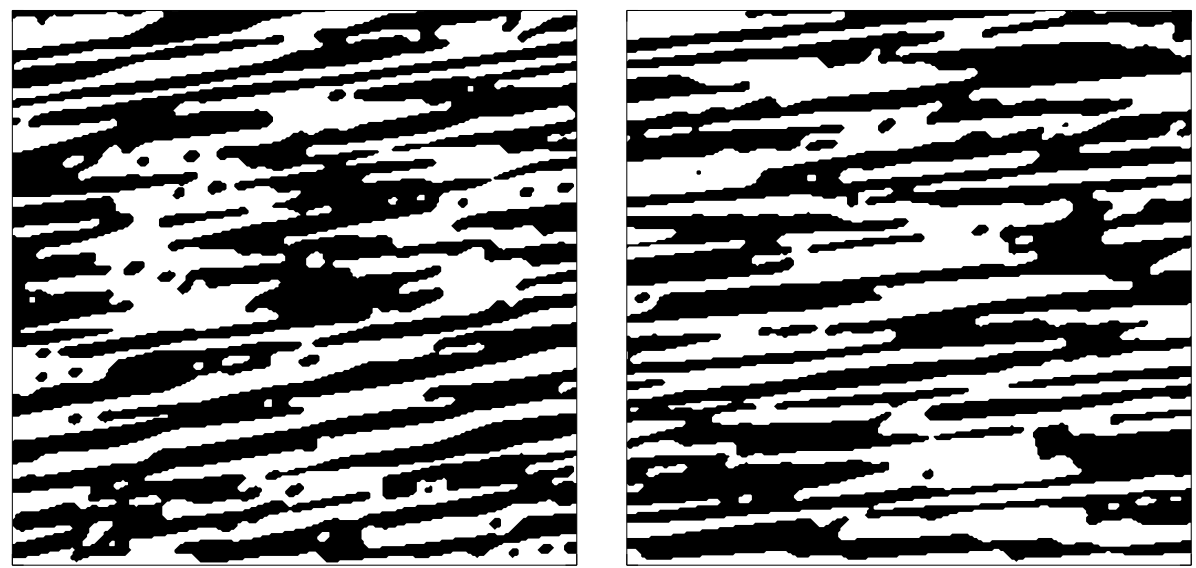

FIG. 1. Configurations at consecutive times of the system in the case with $\lambda=1$. Black/white domains correspond to positive/negative values of the order parameter $\varphi$. A portion of size $256 \times 256$ of the whole lattice is shown. 

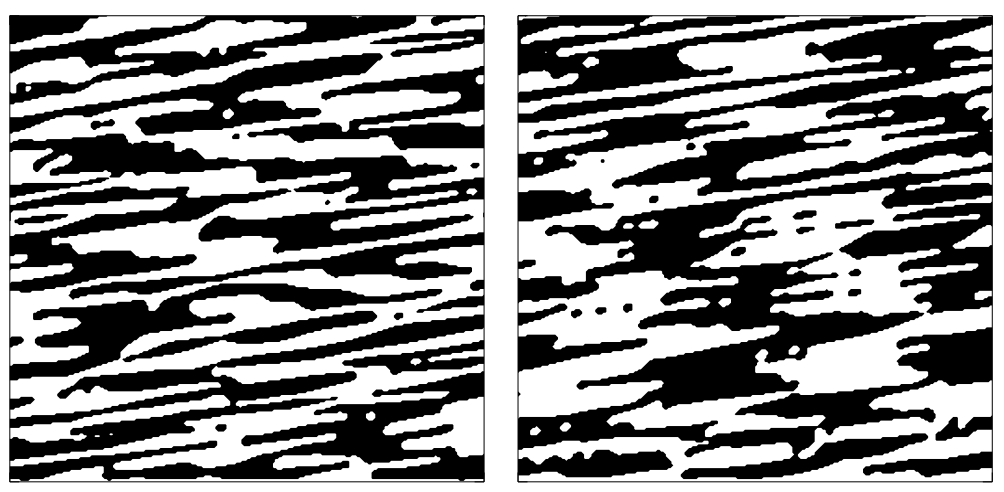

FIG. 2. Configurations at $\dot{\gamma} t=16$ of the system in the cases with $\lambda=0$ (left) and 0.8 (right). Black/white domains correspond to positive/negative values of the order parameter $\varphi$. A portion of size $256 \times 256$ of the whole lattice is shown. 

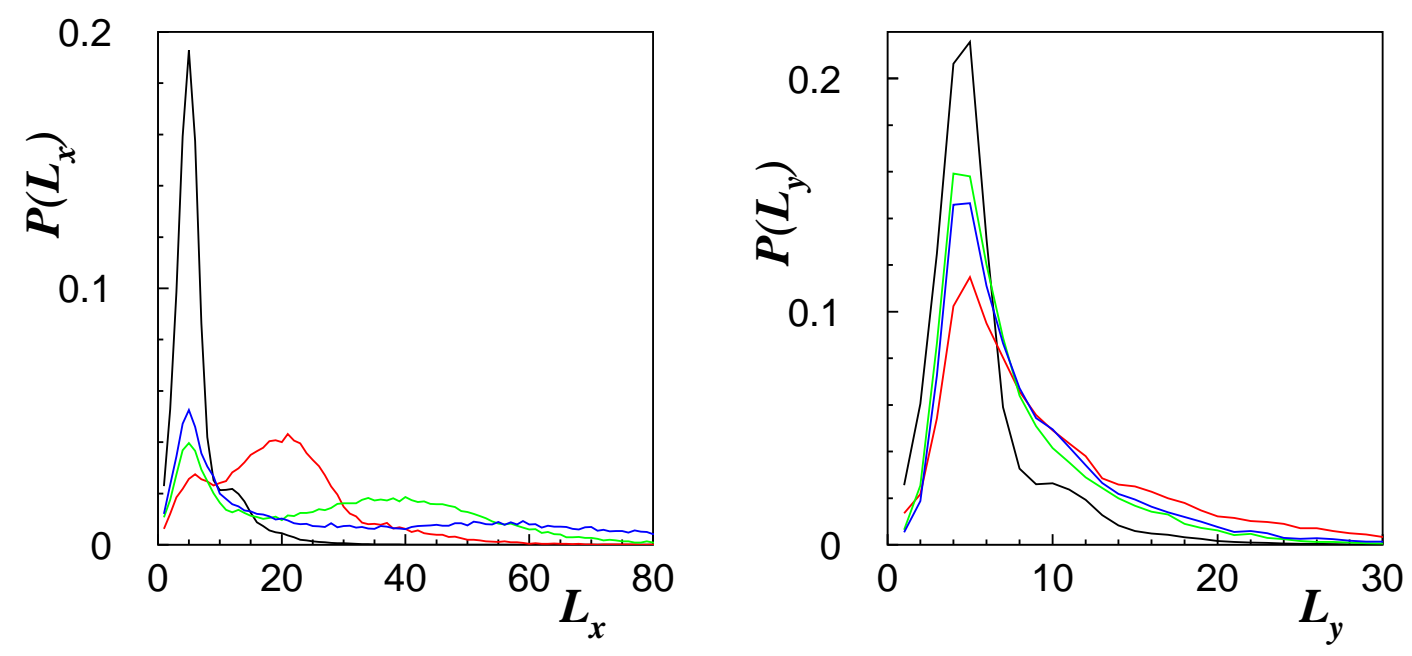

FIG. 3. Probability distribution functions $P$ of domains of length $L_{x}$ (left panel) and $L_{y}$ (right panel) at strains $\dot{\gamma} t=1$ (black line), 7 (red line), 16 (green line), and 24 (blue line) in the case with $\lambda=1$. 


$$
\dot{\gamma} t=12
$$
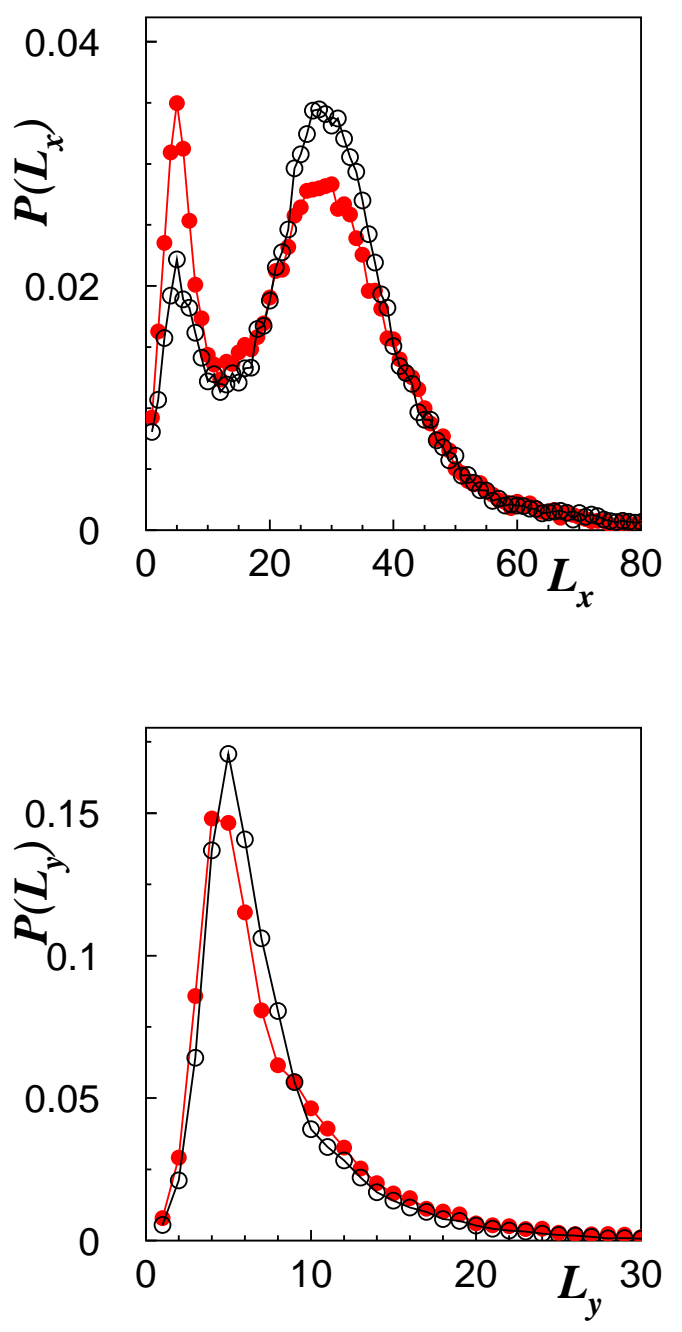

$\dot{\gamma} t=16$
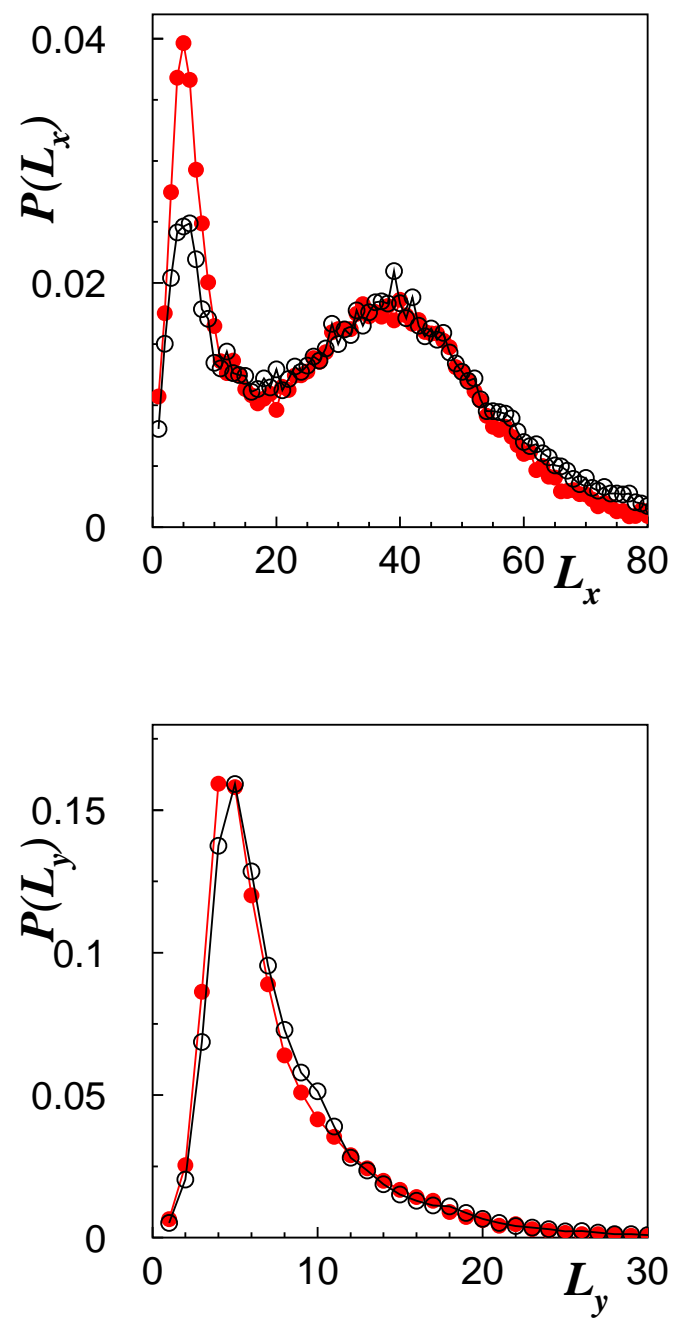

FIG. 4. Probability distribution functions $P$ of domains of length $L_{x}$ (upper panels) and $L_{y}$ (lower panels) at strains $\dot{\gamma} t=12,16$ in the cases with $\lambda=0(\circ), 1(\bullet)$. 


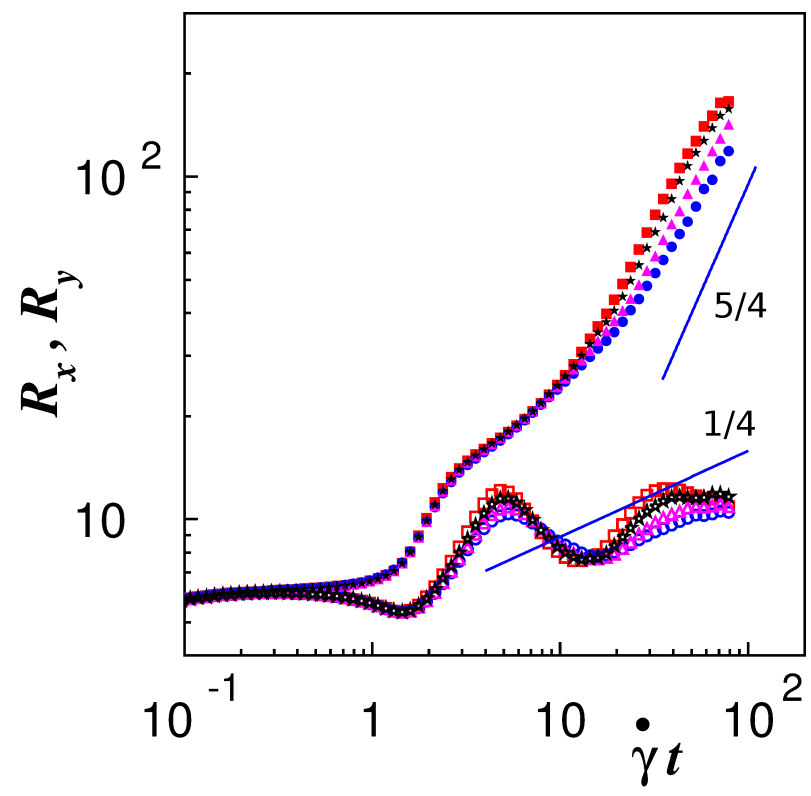

FIG. 5. Average sizes of domains along the flow (filled symbols) and the shear (empty symbols) directions as functions of time for the cases with $\lambda=0(\boldsymbol{\square}), 0.4(\star), 0.8(\boldsymbol{\Delta}), 1(\bullet)$. The lines have slopes $1 / 4$ and $5 / 4$. 


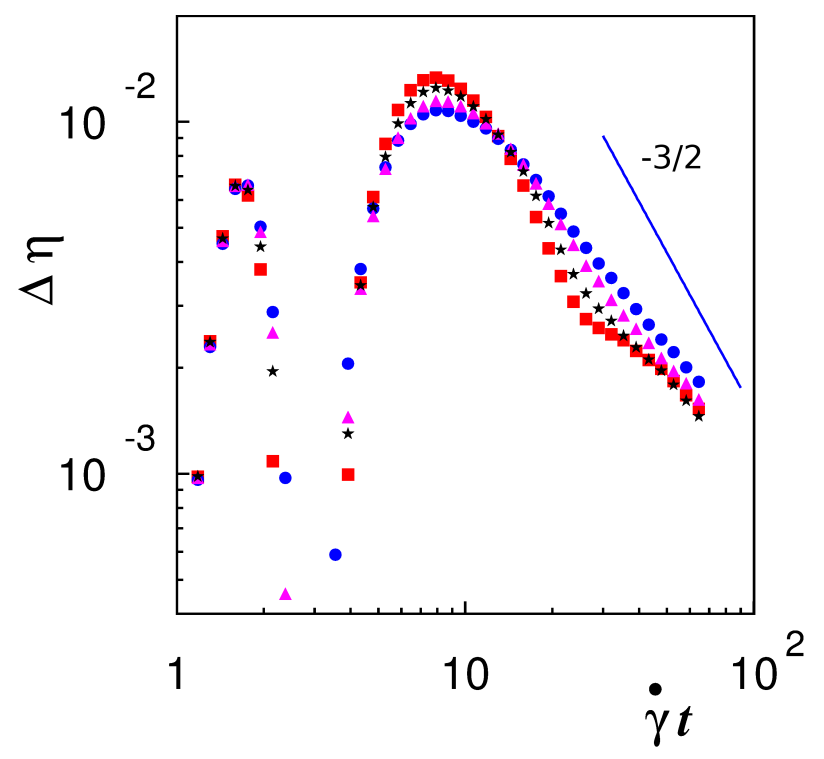

FIG. 6. Excess viscosity as a function of time for the cases with $\lambda=0(\boldsymbol{\square}), 0.4(\star), 0.8(\boldsymbol{\Delta}), 1(\bullet)$. The line has slope $-3 / 2$. 


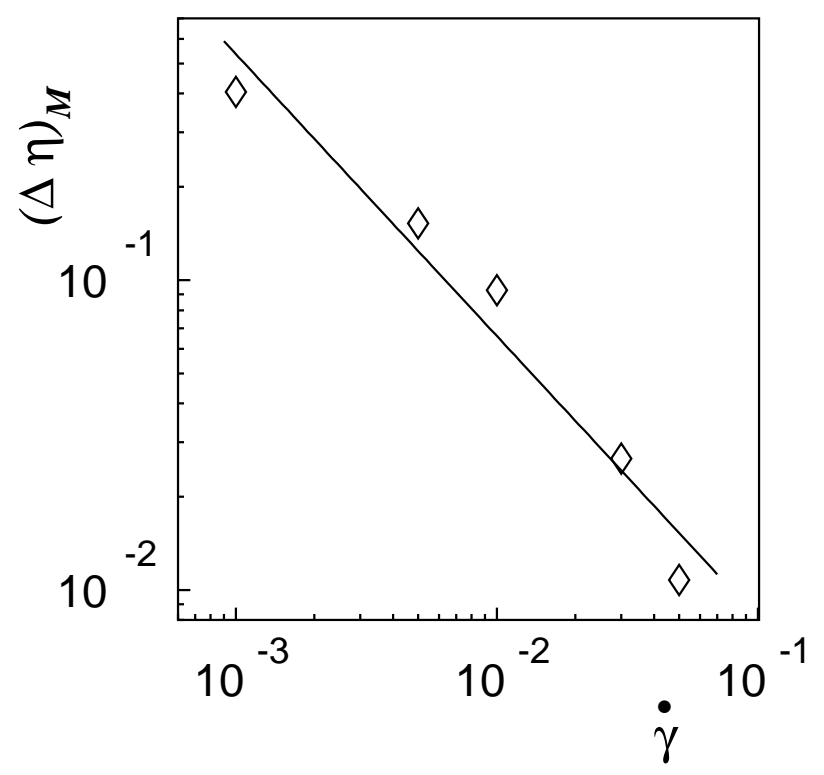

FIG. 7. The maximum of the excess viscosity as a function of the shear rate in the case with $\lambda=1$. The full line is the best fit with slope $-0.91 \pm 0.11$. 